\title{
Mechanisms of fluorescence blinking in semiconductor nanocrystal quantum dots
}

\author{
Jau Tang and R. A. Marcus ${ }^{\text {a) }}$ \\ Noyes Laboratory of Chemical Physics, California Institute of Technology, Pasadena, California 91125
}

(Received 8 April 2005; accepted 12 June 2005; published online 8 August 2005)

\begin{abstract}
The light-induced spectral diffusion and fluorescence intermittency (blinking) of semiconductor nanocrystal quantum dots are investigated theoretically using a diffusion-controlled electron-transfer (DCET) model, where a light-induced one-dimensional diffusion process in energy space is considered. Unlike the conventional electron-transfer reactions with simple exponential kinetics, the model naturally leads to a power-law statistics for the intermittency. We formulate a possible explanation for the spectral broadening and its proportionality to the light energy density, the $-3 / 2$ power law for the blinking statistics of the fluorescence intermittency, the breakdown of the power-law behavior with a bending tail for the "light" periods, a lack of bending tail for the "dark" periods (but would eventually appear at later times), and the dependence of the bending tail on light intensity and temperature. This DCET model predicts a critical time $t_{c}$ (a function of the electronic coupling strength and other quantities), such that for times shorter than $t_{c}$ the exponent for the power law is $-1 / 2$ instead of $-3 / 2$. Quantitative analyses are made of the experimental data on spectral diffusion and on the asymmetric blinking statistics for the "on" and "off" events. Causes for deviation of the exponent from the ideal value of $-3 / 2$ are also discussed. Several fundamental properties are determined from the present experimental data, the diffusion correlation time, the Stokes shift, and a combination of other molecular-based quantities. Specific experiments are suggested to test the model further, extract other molecular properties, and elucidate more details of the light-induced charge-transfer dynamics in quantum dots. (C) 2005 American Institute of Physics. [DOI: 10.1063/1.1993567]
\end{abstract}

\section{INTRODUCTION}

The developments in low-dimensional materials have led to the study of quasi-zero-dimensional systems, known as quantum dots (QDs) or nanocrystals. Nanofabrication techniques have opened a new realm of scientific research. The QDs have unusual optical and electrical properties such as narrower transition linewidth, larger oscillator strength, and tunable band gap covering the visible range. QDs are currently explored extensively in biological tagging applications. ${ }^{1}$ It has also been suggested that QDs may have the potentiality of providing technological applications such as new types of laser, electro-optic modulation devices, highdensity memory devices, and logic gates, among others. ${ }^{2}$

Although the size of quantum dots can be controlled in a reliable way using colloidal nanocrystal preparation methods, and the impact of quantum size effects on optical and electronic properties is understood to some extent, the influence of less controllable surface defects on these properties remains much unknown. For example, the blinking phenomenon $^{3-9}$ (luminescence intermittency) of QDs and its unusual power-law distribution in the histogram of the on-off emission events are still not clearly understood. Intermittency is a phenomenon of a QD showing alternating periods of a bright state (light-induced fluorescence) and a dark state (no emission even under continuous light illumination).

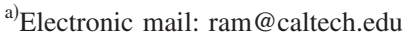

Kuno et $a l .{ }^{8}$ have provided an excellent review of the emission intermittency and examined existing models. They also offered a new charge-tunneling model to treat the phenomenon. Because these models are phenomenological, the physical origin of those hidden variables remains unclear. It is not clearly understood how for such a small QD particle the distribution of the tunneling distance (a hidden variable) can cover five orders of dynamic range in order to account for eight decades ${ }^{5}$ of probability densities in experimental data.

A possible connection of the power-law statistics to the first passage theory in real space or in configuration (energy) space was discussed by Empedocles and Bawendi ${ }^{10}$ and Jung et al. ${ }^{11}$ In the first passage theory, ${ }^{12,13}$ one considers onedimensional (1D) free-space diffusion with an absorbing end point. If the absorption rate at the boundary point is much greater than the in-chain hopping rate, the rate of overall population change follows $t^{-3 / 2}$ power law. ${ }^{12,13}$ The experimental value for the exponent varies among different research groups, some are close to $-1.5,4,5,7,14$ and yet some are slightly higher. ${ }^{6,8,9,15,16}$ A tabulation of the data on the inverse power is given later in Table $\mathrm{V}$.

There are several central questions about QD blinking that one would like to explain on a fundamental basis: (1) Why is the exponent for the power law so close to -1.5 instead of $-1,-2,-3$, or some other value? (2) Why is the power law universal for different QD size, light intensities, and temperatures for the "off" events? (3) Why do the "on"- 
time events follow the same power law as the off events? (4) Why does the power law exist and why does it cover such a large dynamic range (eight decades) of probability density? (5) What mechanism causes only the on event power-law distribution to break down at higher light excitation intensities, longer times, and higher temperatures, ${ }^{4}$ and what mathematical expression best describes the bending tail? (6) What role does spectral diffusion play in QD blinking?

Strong evidence for the correlation between the blinking events with spectral diffusion has been obtained in several studies. ${ }^{17-19}$ The spectral diffusion data and the blinking phenomenon of QDs are analyzed in the present theory using a simple light-driven one-dimensional diffusion model in energy space on parabolic potentials associated with a hole or electron transfer inside shallow traps. The model proposed here provides some molecular answers to the above questions and also permits certain molecular-based properties or combinations of them to be extracted from the data, and suggests further experiments.

In the conventional electron-transfer reactions solvent relaxation is frequently very fast and the electron-transfer reaction is then usually characterized by a single exponential decay. ${ }^{20}$ However, because of very slow energy fluctuations, as clearly evident from the spectral diffusion measurements, the conventional description of an exponential decay is no longer valid.

This model is based on the reaction-diffusion (the "stochastic Liouville") equation for a one-dimensional diffusion process in energy space. A reaction-diffusion approach has been used in the studies ${ }^{21-25}$ of dynamic solvent effects in electron-transfer reactions, and in the present work, slow "structural diffusion" of the QD plays a major role. The fluctuating Stark effect due to charges near or in the QD has been proposed as contributing to the spectral diffusion and intermittency. ${ }^{17}$ The DCET model leads to a -1.5 power-law decay for both on- and off-time statistics. It also leads to the observed breakdown of the power law with a bending tail for the on-time events, ${ }^{4}$ and eventually for the off-time events as well at much longer time. Anomalous diffusion could cause the exponent to deviate from the ideal -1.5 value and will be discussed in Appendix C.

\section{THE PHOTOINDUCED DIFFUSION-CONTROLLED ELECTRON-TRANSFER (DCET) MODEL}

\section{A. Modeling spectral diffusion in QDs}

To examine the spectral diffusion observed in the fluorescence of QDs, we consider a $1 \mathrm{D}$ diffusion on a parabolic free-energy potential representing the excited state $\left|L^{*}\right\rangle$ of the QD and a second parabola representing the ground state of the QD, $|G\rangle$. QDs are known to have a very narrow fluorescence spectrum, but a broad absorption spectrum. As illustrated in Fig. 1(a), this fact indicates that a photoinduced electron-hole pair relaxes rapidly to a band-edge state $\left|L^{*}\right\rangle$ via radiationless processes prior to emission. In Fig. 1(b) parabolic potentials with $q$ as the reaction coordinate are assumed, where $q_{0}$ is the horizontal displacement between the free-energy parabolic potential well for $\left|L^{*}\right\rangle, U_{L}(q)$ $=\kappa q^{2} / 2$, and that for $|G\rangle, U_{G}(q)=\kappa\left(q+q_{0}\right)^{2} / 2-E_{g}$, where $E_{g}$ (a)

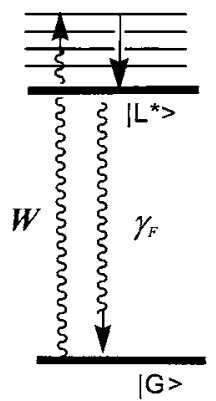

(b)

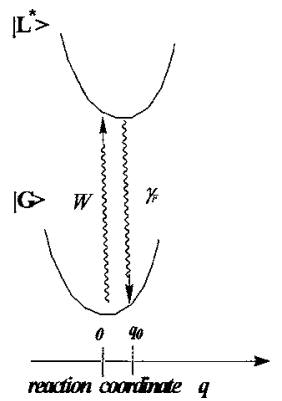

(c)

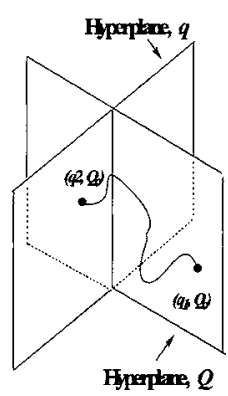

FIG. 1. (a) Schematic diagram for the ground state $|G\rangle$ and the excited light state $\left|L^{*}\right\rangle$. The radiationless decay from the higher excited electronic states to $\left|L^{*}\right\rangle$ is much faster than fluorescence decay $\left(\gamma_{F}\right)$ from $\left|L^{*}\right\rangle$ to $|G\rangle$, and $W$ is the photoexcitation rate. (b) Diffusion on potential of $\left|L^{*}\right\rangle$ and with $q$ as reaction coordinate. (c) Depiction of an event of intermittency and spectral diffusion as random walk in $N$-dimensional space projected onto the hyperplanes of $q$ and $Q$ coordinates, where $Q$ is the reaction coordinate describing electron transfer between the light and dark states.

is the free-energy gap between the minima of these two potential wells. This coordinate $q$ has been used in the past for radiative processes and for discussion of the Franck-Condon principle. $^{26}$

The differential equation for classical diffusion on a harmonic potential is well known ${ }^{27}$ and can be applied here. The rate equations for the probability $f(q, t)$ of finding a QD at $q$ in state $\left|L^{*}\right\rangle$ is given by

$$
\frac{\partial}{\partial t} f(q, t)=D_{1}\left(\frac{\partial^{2}}{\partial q^{2}} f(q, t)+\frac{1}{k \Delta_{1}^{2}} \frac{\partial}{\partial q}\left(f(q, t) \frac{\partial}{\partial q} U_{1}(q)\right)\right),
$$

where $D_{1}$ is the diffusion constant related to $\tau_{1}$, the diffusion correlation time constant by $\tau_{1}=\Delta_{1}^{2} / D_{1}$, and

$$
\kappa \Delta_{1}^{2}=(\hbar \Omega / 2) \operatorname{coth}\left(\hbar \Omega / 2 k_{B} T\right) \approx k_{B} T,
$$

where $k_{B} T$ is the classical $\operatorname{limit}^{25}$ and $\Omega$ is the structural vibration frequency of the QD. According to Empedocles and Bawendi, ${ }^{10}$ the diffusion is light induced and depends on the light intensity and the excess photon energy. Such diffusion on the potential surfaces for $\left|L^{*}\right\rangle$ results in spectral diffusion of the observed spectrum. There are higher electronic states, but there is relaxation to $\left|L^{*}\right\rangle$, the excess energy being released to the lattice also assists diffusion. ${ }^{10}$

For an initial population at any given point $q$ of the reaction coordinate, the probability density $G\left(q, q^{\prime} ; t\right)$ of finding this QD that was initially at $q$ to be at another point $q^{\prime}$ at time $t$ is given by $^{27}$

$$
\begin{aligned}
G\left(q, q^{\prime} ; t\right)= & \frac{1}{\sqrt{2 \pi \Delta_{1}^{2}\left(1-\exp \left(-2 t / \tau_{1}\right)\right)}} \\
& \times \exp \left[-\frac{\left(q-q^{\prime} \exp \left(-t / \tau_{1}\right)\right)^{2}}{2 \Delta_{1}^{2}\left(1-\exp \left(-2 t / \tau_{1}\right)\right)}\right] .
\end{aligned}
$$

One can now determine the time evolution of the spectral linewidth for the radiative transition from $\left|L^{*}\right\rangle$ to $|G\rangle$. By the Franck-Condon principle, the spectral emission energy is 
$U_{L}(q)-U_{G}(q)$, i.e., $E_{g}-\kappa q_{0}^{2} / 2+\kappa q q_{0}$. The change in coordinate $q$ describes a spectral diffusion in the fluorescence of $\left|L^{*}\right\rangle$.

From the time evolution of $f(q, t)$ and Eq. (2), the second moment of the emission spectrum is

$$
\begin{aligned}
\sigma^{2}(t) & =\left\langle(\Delta U(q)-\langle\Delta U(q)\rangle)^{2}\right\rangle \\
& =\int_{-\infty}^{\infty} d q\left(\kappa q q_{0}\right)^{2} G(q, q ; t) \\
& =2 \kappa \Lambda \Delta_{1}^{2}\left[1-\exp \left(-t / \tau_{1}\right)\right],
\end{aligned}
$$

where $\Delta U(q)=U_{L}(q)-U_{G}(q), \Lambda=\kappa q_{0}^{2} / 2$ is the "excitation reorganization energy," $2 \Lambda$ is the Stokes shift, and $\kappa \Delta_{1}^{2}$ is given by Eq. (1b).

\section{B. Modeling intermittency in QDs}

Unlike the static origin assumed in a distributed rate model, the power-law decay in a QD is assumed here to have a dynamic origin resulting in energy fluctuations and diffusion. In the present study, nonadiabatic electron-transfer theory with diffusion-controlled reactions is explored. In this case, diffusive processes occur along free-energy potentials with a sink at the energy level crossing $Q=Q_{c}$ for the electron transfer between the light and the dark states. The electrostatic properties of the dark QDs have been studied by Krauss and Brus $^{28}$ and Cherniavskaya et al. ${ }^{29}$

To treat intermittency phenomenon, ${ }^{2-8}$ a four-level model is proposed and the reaction-diffusion equation is solved. The model consists of two neutral states and two charge-separated states. $|D\rangle$ describes a charge-separated state with a charge in the core and a countercharge assumed to be trapped in surface states ${ }^{30,31}$ just below the edge of the quasiconduction band or just above the edge of the quasivalence band. $\left|D^{*}\right\rangle$ represents the excited charge-separated state of $|D\rangle$ with an additional exciton. Figure 2(a) schematically represents a total energy for each state, where the transition between $\left|L^{*}\right\rangle$ and $|G\rangle$ is the bottleneck process responsible for intermittency. The spectral diffusion for the emission from $\left|L^{*}\right\rangle$ to $|G\rangle$ was described in Sec. II A. On a quartz substrate used in most experiments, photoemission from $\left|D^{*}\right\rangle$ and $|D\rangle$ is quenched by an Auger process, so the chargeseparated QDs appear dark and only the neutral QDs emit photons. ${ }^{2-8}$ Photoemission from $\left|D^{*}\right\rangle$ to $|D\rangle$ for QDs was observed on a rough gold surface by Shimizu et al. ${ }^{7}$ and this fact indicates that the surface-enhanced radiative rate has become comparable with the rate of the Auger nonradiative process previously responsible for the QD being dark. The binary jump between two emission tracks of a QD on a rough gold surface ${ }^{7}$ indicates forward and backward transitions between $\left|L^{*}\right\rangle$ and $|D\rangle$, and the emission tracks represent the vertical energy difference between $\left|L^{*}\right\rangle$ and $|G\rangle$ on one hand, and $\left|D^{*}\right\rangle$ and $|D\rangle$ on the other.

A reaction coordinate $Q$ is defined for electron transfer as the vertical difference between free-energy parabolas of the neutral light state $\left|L^{*}\right\rangle$, and the low-lying chargeseparated (dark) state $|D\rangle$ resulting from an electron transfer (a)

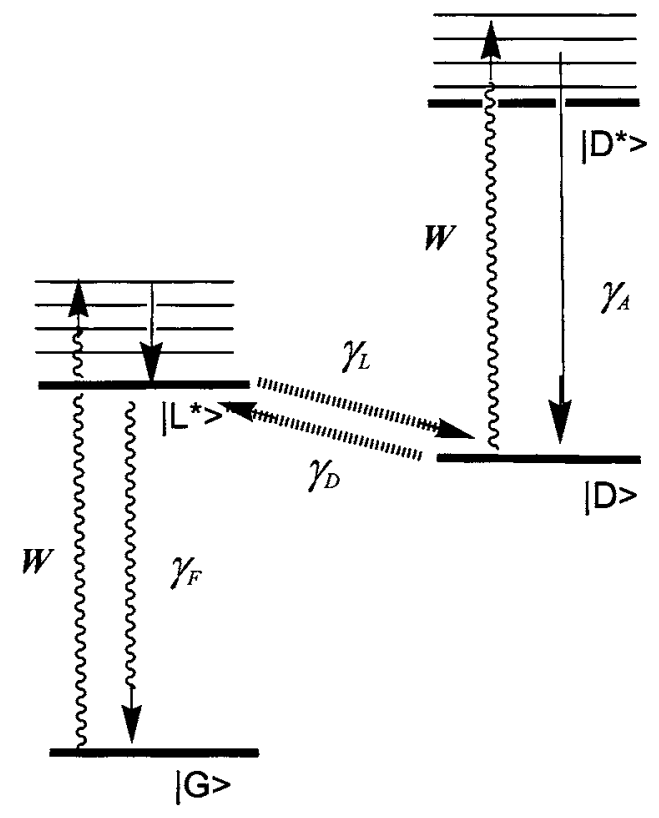

(b)

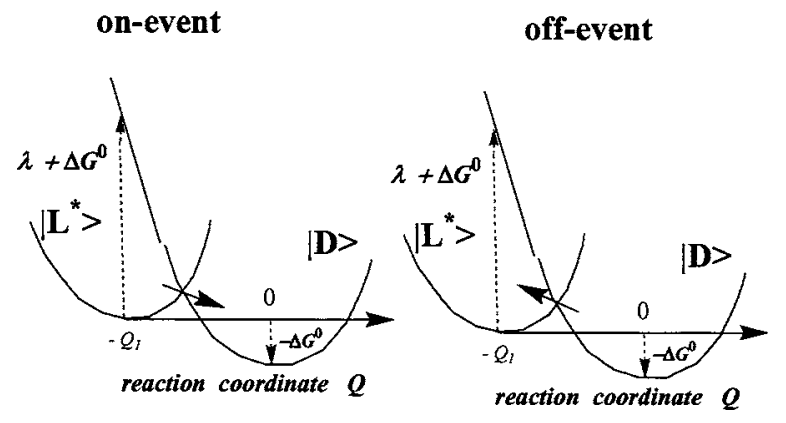

FIG. 2. (a) Schematic diagram for the DCET model. The transition between $\left|L^{*}\right\rangle$ and $|D\rangle$ represents the bottleneck charge separation and recombination processes. Decay from $\left|D^{*}\right\rangle$ to $|D\rangle$ is caused by radiationless Auger processes $\left(\gamma_{A}\right)$ on ordinary substrates, but can become radiative on gold surface. (b) Diffusion on the parabolic potential surfaces for $\left|L^{*}\right\rangle$ and $|D\rangle$ across a sink at the energy-level crossing governs the intermittency phenomenon.

is introduced. This $Q$ has been used earlier by Marcus ${ }^{32}$ and Warshel $l^{33}$ as a reaction coordinate for electron-transfer reactions.

As illustrated in Fig. 1(c), at a birth of the on state a QD jumps from the off state at the energy level crossing at $Q$ $=Q_{c}$. This one-dimensional coordinate $Q$ describes an ensemble of points in a hyperplane in $N$-dimensional space. ${ }^{32}$ For each event of a light or dark period, $Q$ starts and ends at $Q_{c}$, the crossing point of the parabolas for $\left|L^{*}\right\rangle$ and $|D\rangle$. The coordinates $q$ and $Q$ are discussed further in Appendix B.

To calculate the blinking statistics for, say, the on events, one needs to evaluate the lifetime of the distribution for a QD that stays in a light state. The initial condition for motion in the light or dark state is a delta-function population at the crossing. As illustrated in Fig. 2(b), the potentials are defined as $U_{1}(Q)=\kappa_{E}\left(Q+Q_{1}\right)^{2} / 2$ and $U_{2}(Q)=\Delta G^{0}+\kappa_{E} Q^{2} / 2$, where $\lambda=\kappa_{E} Q_{1}^{2} / 2$ is the well-known reorganization energy for electron transfer and $\Delta G^{0}$ is the free-energy gap for electron transfer between $|D\rangle$ and $\left|L^{*}\right\rangle$. The energy-level crossing (the 
sink) occurs at $Q_{c}=\left(\Delta G^{0}-\lambda\right) / \sqrt{2 \kappa_{E} \lambda}$. The $q$ and $Q$ are different projections from an $N$-dimensional surface onto only a single coordinate, each of which describes an $(N-1)$-dimensional ensemble. The force constant $\kappa_{E}$ in electron transfer is expected to be different from the $\kappa$ involved in spectral diffusion, since the two coordinates represent a differently weighted linear combination of $N-1$ individual oscillators.

The reaction rate is written in terms of a nonadiabatic electron-transfer rate expression represented by a deltafunction term in Eq. (4a) and (4b) below. The distribution of lifetimes of a QD to stay continuously in the neutral state is calculated from the solution of this equation. According to Eq. (A2) in Appendix A, the time evolution of population distribution $\rho_{11}(Q, t)$ on potential $U_{1}(Q)$ and a sink at $Q_{c}$ satisfies

$$
\begin{aligned}
\frac{\partial}{\partial t} \rho_{11}(Q, t)= & \frac{1}{\tau_{1}}\left(\Delta_{1}^{2} \frac{\partial^{2}}{\partial Q^{2}}+\left(1+\left(Q+Q_{1}\right) \frac{\partial}{\partial Q}\right)\right) \rho_{11}(Q, t) \\
& -\frac{2 \pi\left|V_{12}\right|^{2}}{\hbar} \delta\left(U_{1}(Q)-U_{2}(Q)\right) \rho_{11}(Q, t), \quad(4 a)
\end{aligned}
$$

where $\Delta_{1}^{2} / \tau_{1} \equiv D_{1}, V_{12}$ is the electronic coupling between states $\left|L^{*}\right\rangle$ and $|D\rangle$, and the initial condition $\rho_{11}(Q, 0)=\delta(Q$ $-Q_{c}$ ).

Similarly, for a single dark QD on $U_{2}(Q)$, the population distribution $\rho_{22}(Q, t)$ satisfies

$$
\begin{aligned}
\frac{\partial}{\partial t} \rho_{22}(Q, t)= & \frac{1}{\tau_{2}}\left(\Delta_{2}^{2} \frac{\partial^{2}}{\partial Q^{2}}+\left(1+Q \frac{\partial}{\partial Q}\right)\right) \rho_{22}(Q, t) \\
& -\frac{2 \pi\left|V_{21}\right|^{2}}{\hbar} \delta\left(U_{1}(Q)-U_{2}(Q)\right) \rho_{22}(Q, t),
\end{aligned}
$$

with the initial condition $\rho_{22}(Q, 0)=\delta\left(Q-Q_{c}\right)$. Equations (4a) and (4b) describe a 1D reaction-diffusion on parabolic free-energy potentials with a reaction sink at their intersection.

The blinking statistics $P_{\text {on }}(t)$ for the on events of the neutral QDs (or $P_{\text {off }}(t)$ for the off events of the dark QDs) are defined as the waiting-time distribution function for a QD that is initially in the neutral "light" states $|G\rangle$ and $\left|L^{*}\right\rangle$ (or charge-separated "dark" states $\left|D^{*}\right\rangle$ and $|D\rangle$ ) and is turned into a dark state (or light state) between $t$ and $t+d t$ per unit $d t$.

The Green function method ${ }^{34}$ can be used to solve for $P(t)$ from Eq. (4a) or (4b). Its explicit approximate form is given by Eq. (A11). To obtain Eqs. (5a)-(5c), (6a), and (6b) below we introduce the approximation (Appendix A) that $t$ $\ll \tau_{k}$. If $t$ is much smaller than the critical time constant $t_{c, k}$, Eq. (A11) can be approximated by

$$
P_{k}(t) \approx \frac{1}{\sqrt{\pi t_{c, k}}} t^{-1 / 2} \quad \text { if } t \ll t_{c, k},
$$

and if, instead, $t$ is much longer,

$$
P_{k}(t) \approx \frac{\sqrt{t_{c, k}}}{2 \sqrt{\pi}} t^{-3 / 2} \quad \text { if } t \gg t_{c, k},
$$

where

$$
t_{c, k} \equiv 2 \kappa_{E} \lambda\left(\frac{\hbar \Delta_{k}}{\left|V_{k}\right|^{2} \pi \sqrt{\tau_{k}}}\right)^{2}
$$

For time $t$ comparable to the saturation time $\mathrm{I} / \Gamma_{k}$, but shorter than the effective diffusion time constant $\tau_{k}, P_{k}(t)$ for the blinking statistics is obtained in Eq. (A11),

$$
P_{k}(t) \approx \frac{\sqrt{t_{c, k}}}{2 \sqrt{\pi}} t^{-3 / 2} \exp \left(-\Gamma_{k} t\right), \quad k=1 \text { (on) } \quad \text { or } 2 \text { (off) },
$$

where

$$
\Gamma_{\text {on }}=\frac{\left(\lambda+\Delta G^{0}\right)^{2}}{8 \tau_{1} \kappa_{E} \lambda \Delta_{1}^{2}}, \quad \Gamma_{\text {off }}=\frac{\left(\lambda-\Delta G^{0}\right)^{2}}{8 \tau_{2} \kappa_{E} \lambda \Delta_{2}^{2}} .
$$

Equations (5a)-(5c), (6a), and (6b) are key results for the intermittency in the present work, and Eq. (3) is the key equation for spectral diffusion.

\section{DISCUSSION OF EQUATIONS}

In the present model the role of the light absorption in spectral diffusion is to catalyze the rate of attainment of a thermal equilibrium distribution of $q$ (i.e., change $\tau_{k}$ rather than create an athermal distribution). An experiment which can distinguish the two effects is described later. Since $\kappa_{E} \Delta_{1}^{2}$ is given by Eq. (1b), the $\sigma^{2}(t=\infty)$ in Eq. (3) is independent of the light intensity. That is, ultimately, the light absorption leads to the attainment of a thermal equilibrium, whose position is independent of light intensity. Since diffusion is light driven and $1 / \tau_{1}$ is proportional to the light intensity or $W$, Eq. (3) implies that the second moment has a universal proportionality to the combined variable $W t$, or, equivalently, to the light energy density.

The critical time $t_{c, k}$ appearing in Eqs. (5a)-(5c) and dividing the two power-law regimes has an interesting physical interpretation: Equation (6a) can be rewritten as

$$
\frac{2 \pi}{\hbar}\left|V_{k}\right|^{2} t_{c, k}=\sqrt{4 D_{k} t_{c, k}} \times \sqrt{2 \kappa_{E} \lambda},
$$

with $D_{k}=\Delta_{k}^{2} / \tau_{k}$ and $\sqrt{2 \kappa_{E} \lambda}$ is related to the slope of the potential at the crossing. The right-hand side of Eq. (7a) is approximately the energy spread in $q$ space at time $t_{c, k}$ due to diffusion. The left-hand side, when multiplied by a density of states, is the population depletion by the sink in time $t_{c, k}$. The reciprocal of the right side serves as that state's density, and so this equation means that $t_{c, k}$ is the time in which the population has largely been depleted due to disappearance into the sink. What is observed in the intermittency studies, since $t$ $\gg t_{c, k}$, is only a very small residual survival of a light (or for $k=2$, dark) QD state.

The power law of $t^{-1 / 2}$ for $t \ll t_{c, k}$ can be understood in physical terms by noting that the probability decay rate, $\partial P(t) / \partial t$, equals the product of $2 \pi\left|V_{k}\right|^{2} / \hbar$, the density of states, and $\rho_{k k}$. The density of states near $Q_{c}$ at time $t$ is approximately $\sqrt{2 \kappa_{E} \lambda} / \sqrt{4 D_{k} t}$, and so this probability decay rate varies as $t^{-1 / 2}$. For times longer than $t_{c, k}$, the population diffusion gradient has been set up at the sink at $Q_{c}$, and a 
population varies as $t^{-1 / 2}$, and so the rate of change of this population $\partial P / \partial t$ in this now diffusion-controlled regime is proportional to $t^{-3 / 2}$.

The physical significance of $\Gamma_{k}$ in Eq. (7b) can also be seen by using the equivalent for $\Gamma_{k}$ given by Eq. (A9a) and (A9b), $\Gamma_{k}=\left(Q_{c}-x_{0, k}\right)^{2} / 4 \tau_{k} \Delta_{k}^{2}$, and Eq. (A8) for the Green function. The mean-square displacement from the crossing point at equilibrium $(t=\infty),\left\langle\left(Q(\infty)-Q_{c}\right)^{2}\right\rangle$, is related to $\Gamma_{k} \tau_{k}$ by

$$
\Gamma_{k} \tau_{k}=\frac{\left\langle\left(Q(\infty)-Q_{c}\right)^{2}\right\rangle}{4 \Delta_{k}^{2}}-\frac{1}{4}=\frac{E_{a, k}}{2 k_{B} T},
$$

where $E_{A, k}$ defined in Eq. (A9b) is the energy difference between the crossing point and the bottom of the potential well $U_{k}(Q)$. Accordingly, the damping factor $\Gamma_{k}$ reflects the additional loss rate in surviving probability, where the potential crossing does not occur at the bottom of the potential well.

If the potential $U_{k}(Q)$ were flat, the right-hand side of Eq. (7b) would vanish, and with it the exponential decay term would vanish since $\exp \left(-\Gamma_{k} t\right)=1$ at $\Gamma_{k}=0$. Thus, the exponential decay is associated with the effect of the potential $U_{k}$ causing the residual population to move away from $Q_{c}$ more rapidly than it otherwise would, so causing the rate of population decay to drop more precipitously.

We consider next the differences in $\Gamma_{k}$ for $k=1$ and 2. In Eq. (6b) $\Gamma_{k}$ is proportional to $1 / \tau_{k}$. As compared to the fluorescence decay time $(\sim 10 \mathrm{~ns})^{35}$ of the light state, Auger relaxation $(\sim 30 \mathrm{ps})$ in the dark state is fast, ${ }^{36}$ and $\tau_{2}$ could be substantially longer. A smaller $\Gamma_{2}$ could be attributed to a larger $\tau_{2}$ than $\tau_{1}$, according to Chung and Bawendi ${ }^{37}$ for their observation of a very slow backward reaction. The other controlling factor for $\Gamma_{k}$, namely, $\left(\lambda \pm \Delta G^{0}\right)^{2}$, is the size of $\Delta G^{0}$. A positive value for $\Delta G^{0}$ could also lead to a smaller bending.

The observable number of decades for the $t^{-3 / 2}$ power law is limited both above and below; in the present model the upper time limit is dictated by the factor $1 / \Gamma_{k}$, which is 10-100 s for the light state, depending on light intensity, temperature, etc. The lower limit is dictated by the critical time $t_{c, k}$ in Eq. (6a). This transition of the power law in Fig. 3(a) has not been observed in $0.1 \mathrm{~ms}$, which is currently the shortest time used for the power-law data from Kuno et al. ${ }^{8}$ and Shimizu et al. ${ }^{5}$ Accordingly, this critical time must be shorter than $0.1 \mathrm{~ms}$. The regimes of $-1 / 2$ and $-3 / 2$ are illustrated in the numerical calculations given in Fig. 3(a). A power law different from $-1 / 2$ and $-3 / 2$ can arise if anomalous diffusion is present due to a distribution of the diffusion correlation times and will be discussed in Appendix C.

\section{ANALYSIS OF THE EXPERIMENTAL DATA}

\section{A. Analysis of the spectral diffusion data}

The experimental observations by Empedocles and Bawendi ${ }^{10}$ showed the intimate correlation between the spectral diffusion and the intermittency, where a QD changes in time between the charge-separated dark state and the neutral light state. The spectral peak of photoluminescence in QDs (a)

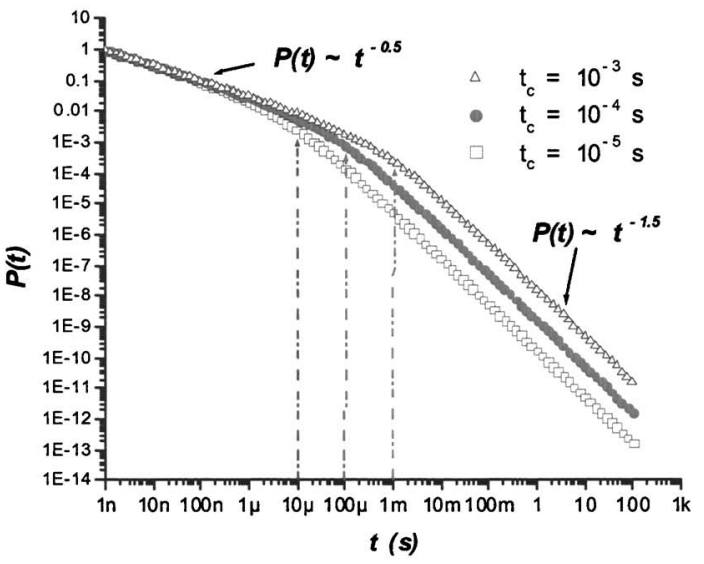

(b)

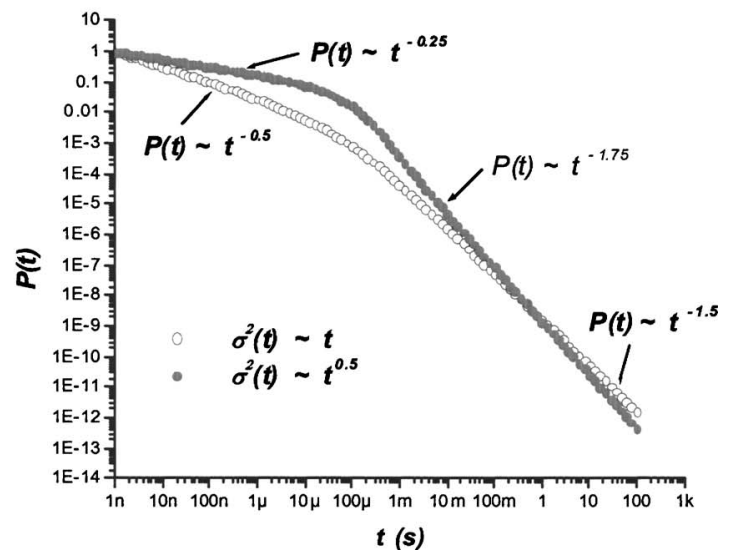

FIG. 3. (a) $P(t)$ of Eq. (A11) for various values of the critical time $t_{c}$ for the ordinary diffusion with $\sigma_{2}(t) \sim t$. The change of the power-law behavior with a different exponent occurs at $t=t_{c}$, and the transition point is indicated by the vertical dot line for each case. (b) The blinking statistics $P(t)$ for two cases with the second moment of spectral broadening $\sigma^{2}(t) \sim t$ vs $\sigma^{2}(t)$ $\sim t^{1 / 2}$. In the first normal diffusion case, $P(t) \sim t^{-1 / 2}$ at shorter time and $P(t) \sim t^{-3 / 2}$ at longer time. In the second anomalous diffusion case, at shorter time $P(t) \sim t^{-1 / 4}$, but changes into $t^{-7 / 4}$ later.

changes stochastically and the linewidth becomes broadened in 10-100 s and increases with light intensity. ${ }^{10}$

According to the estimate of Osborne et al. ${ }^{38}$ the absorption cross section for a typical QD is of the order of $10^{-15} \mathrm{~cm}^{2} \mathrm{eV}$. With an excitation intensity of $400 \mathrm{~W} / \mathrm{cm}^{2}$, the time interval between absorption of photons is $1 / W$ $\sim 1 \mu \mathrm{s}$, whereas the fluorescent lifetime for an electron-hole pair in a QD is of the order of $10 \mathrm{~ns}^{34}$ Accordingly, $W$ $\ll \gamma_{F}$ for most experiments with an intensity below $2 \mathrm{~kW} / \mathrm{cm}^{2}$.

The linewidth data of Empedocles and Bawendi ${ }^{10}$ are plotted in Figs. 4(a) and 4(b). They show the dependence of the second moment on time and excitation energy. A factor of $(1 / 8) \ln 2$ was used in relating the second moment to the square of the linewidth. The dependence of the second moment on energy density (the product of time and excitation intensity) is illustrated in Fig. 5(a). This universal dependence on energy density was experimentally observed and pointed out by Empedocles and Bawendi. ${ }^{10}$ The fitted curves are shown as dotted lines in Figs. 4(a), 4(b), and 5(a). The parameters in Table I are obtained by fitting Figs. 4(a) and 
(a)

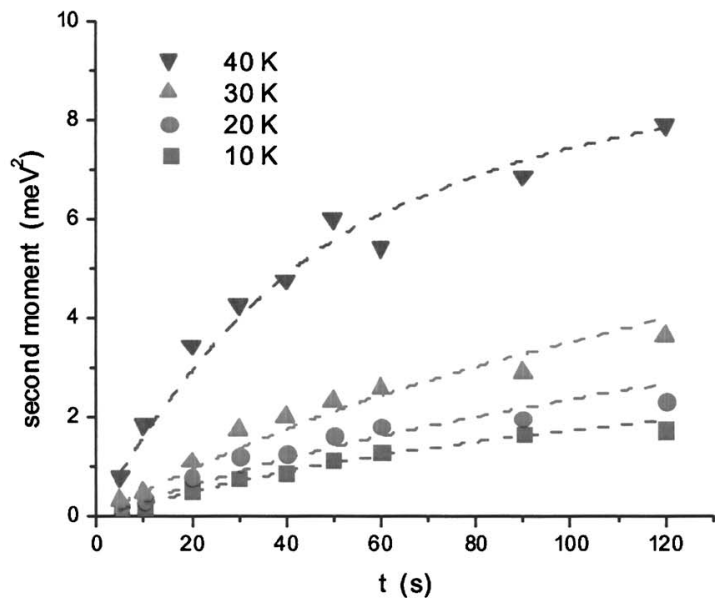

(b)

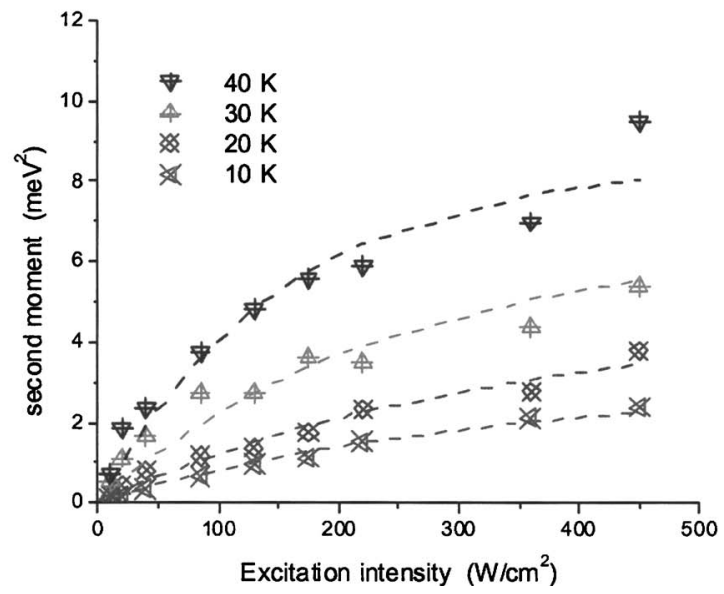

FIG. 4. (a) The time dependence of the spectral second moment at various temperatures with excitation intensity at $85 \mathrm{~W} / \mathrm{cm}^{2}$. (b) The dependence of the spectral second moment (at $t_{\mathrm{obs}}=30 \mathrm{~s}$ ) on excitation intensity $I$. The dot curves represent the fitted data with the fitted parameters and equations listed in Table I.

4(b) with $\sigma^{2}(t)=2 \kappa \Lambda \Delta_{1}^{2}\left[1-\exp \left(-2 t / \tau_{1}\right)\right]$ for an excitation intensity $I$ of $85 \mathrm{~W} / \mathrm{cm}^{2}$. Here the light-driven diffusion model is assumed with $1 / \tau_{1} \equiv W / \gamma_{F} \tau_{1,0}$. In the dark, there is an intrinsic, but small diffusion component that is neglected in this work. Because the curve at $40 \mathrm{~K}$ approaches saturation faster, the fitting of $\kappa \Lambda \Delta_{1}^{2}$ is more reliable. Accuracy can be improved if more data near saturation were available. For curve fitting at three lower temperatures, the constraint of $\kappa \Delta_{1}^{2}=(\hbar \Omega / 2) \operatorname{coth}\left(\hbar \Omega / 2 k_{B} T\right)$ was imposed. The parameters $\kappa \Lambda \Delta_{1}^{2}$ and $I_{0}$ for Fig. 4(b) are given with standard deviations in parentheses, using $\sigma^{2}(t)=2 \kappa \Lambda \Delta_{1}^{2}\left[1-\exp \left(-I / I_{0}\right)\right]$ for $t_{\text {obs }}$ $=30 \mathrm{~s}$, where $I_{0}=\gamma_{F} \tau_{1,0} / 2 t_{\mathrm{obs}} \sigma_{\mathrm{abs}}$, with $\sigma_{\mathrm{abs}}$ as the cross sec-

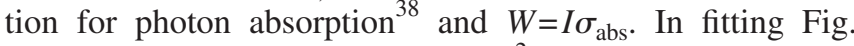
4(b), the asymptotic value of $2 \kappa \Lambda \Delta_{1}^{2}$ at a given temperature was obtained earlier from Fig. 4(a). The parameters in Table II are obtained by fitting the data in Fig. 5(a) using this formula for $\sigma^{2}$, with $I / I_{0}$ replaced by $J / J_{0}$, where $J$ represents the energy density $(I \times t)$ and $J_{0}=\gamma_{F} \tau_{1.0} / 2 \sigma_{\text {abs }}$.

From Fig. 4(a) and Table I for excitation intensity at $85 \mathrm{~W} / \mathrm{cm}^{2}$, the time constant $\tau_{1}$ and $2 \kappa \Lambda \Delta_{1}^{2}$ for the spectral diffusion have been determined, with $\kappa \Delta_{1}^{2}$ being given by Eq. (a)

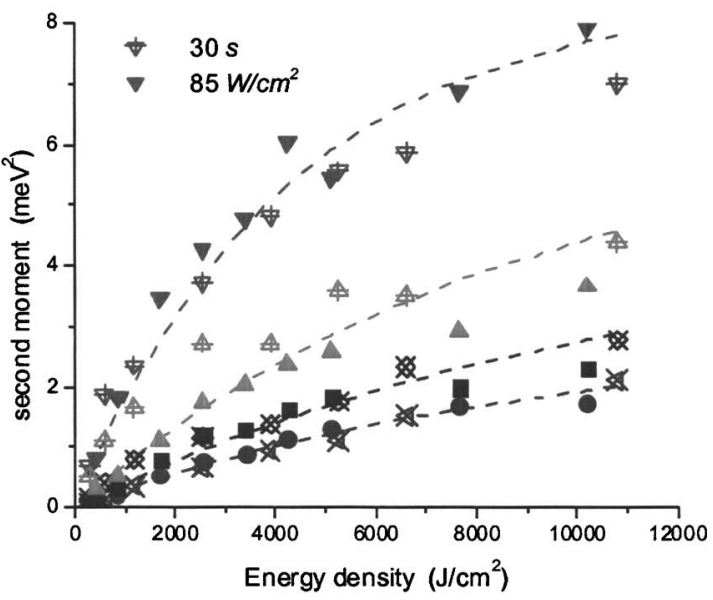

(b)

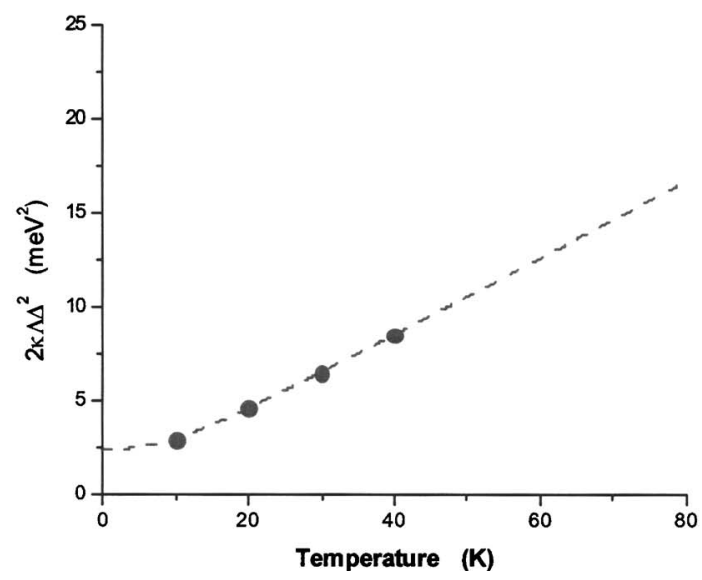

FIG. 5. (a) The dependence of the second moment on energy density $J(I$ $\times t$ ) showing the energy density as a universal variable. The dot curves represent the fitted data using $2 \kappa \Lambda \Delta_{1}^{2}\left[1-\exp \left(-J / J_{0}\right)\right]$, where $2 \kappa \Lambda \Delta_{1}^{2}$ and $J_{0}$ are listed in Table II. (b) The temperature dependence of $2 \kappa \Lambda \Delta_{1}^{2}$ in Table II. With quantum correction for $2 \kappa \Lambda \Delta_{1}^{2}=\Lambda \hbar \Omega \operatorname{coth}\left(\hbar \Omega / 2 k_{B} T\right)$, one finds $\Lambda \hbar \Omega=2.4 \mathrm{meV}^{2}$ and $\hbar \Omega=2 \mathrm{meV}$. Because $\hbar \Omega$ is small, classical description is valid above $40 \mathrm{~K}$.

(1b). From the analysis of Figs. 4(b) and 5(a), it can be seen that the second moment increases with light intensity and energy density. In the high-temperature limit, one expects $\Delta_{1}^{2}$ to increase linearly with temperature. Based on Tables I and II, as temperature increases $2 \kappa \Lambda \Delta_{1}^{2}$ increases and $\tau_{1}$ is expected to decrease. Thereby, the diffusion constant $D_{1}$ (i.e., $\Delta_{1}^{2} / \tau_{1}$ ) will also increase. The temperature dependence of $2 \kappa \Lambda \Delta_{1}^{2}$ is illustrated in Fig. 5(b). In the fitting of Figs. 4(a) and $4(\mathrm{~b})$ to the quantum form of Eq. (1b), $\hbar \Omega$ is estimated to be about $2 \mathrm{meV}$. At $40 \mathrm{~K}, 2 \kappa \Lambda \Delta_{1}^{2}=8.5 \mathrm{meV}^{2}$. From these results one can use $\kappa \Delta_{1}^{2} \approx k_{B} T$ at $40 \mathrm{~K}$ or use Eq. (1b) and the $\Omega$ estimated from the fit in Fig. 2 to estimate the Stokes shift $2 \Lambda$ to be about $2.4 \mathrm{meV}$. This value is in good agreement with the result using zero-phonon line measurements ${ }^{39}$ and theoretical estimate. ${ }^{40}$

Empedocles and Bawendi ${ }^{10}$ observed that the reduction of the photon energy by $250 \mathrm{meV}$ reduced the spectral diffusion by $25 \% .{ }^{10}$ Further experimental study can determine whether this increase occurs in the transient part of $\sigma^{2}(t)$ or in the saturated part $\sigma^{2}(\infty)$, or both. If it is observed only in 
TABLE I. The fitted parameters $2 \kappa \Lambda \Delta_{1}^{2}, \tau_{1}$, and $I_{0}\left(I_{0}=\gamma_{F} \tau_{1,0} / 2 t_{\text {obs }} \sigma_{\text {abs }}\right)$ with enclosed standard deviations for Fig. 4(a) using $\sigma^{2}=2 \kappa \Lambda \Delta_{1}^{2}[1$ $\left.-\exp \left(-2 t / \tau_{1}\right)\right]$ and Fig. 4(b) using $\sigma^{2}=2 \kappa \Lambda \Delta_{1}^{2}\left[1-\exp \left(-I / I_{0}\right)\right]$. The values of $2 \kappa \Lambda \Delta_{1}^{2}$ for Figs. 4(b) and 5(a) were obtained from the fitting of Fig. 4(a) and only $I_{0}$ 's were fitted. Because saturation of the second moment has not been reached at $120 \mathrm{~s}$ for data below $40 \mathrm{~K}$, in the fitting a constraint of $\kappa \Delta_{1}^{2}=(\hbar \Omega / 2) \operatorname{coth}\left(\hbar \Omega / 2 k_{B} T\right)$ was imposed. The actual errors in values of $\tau_{1}$ below $40 \mathrm{~K}$ are large and can be reduced if longer-time data become available.

\begin{tabular}{|c|c|c|}
\hline (K) & $\begin{array}{c}\text { Fig. } 4(\mathrm{a}) \\
\left(W=85 \mathrm{~W} / \mathrm{cm}^{2}\right)\end{array}$ & $\begin{array}{l}\text { Fig. } 4(\mathrm{~b}) \\
\left(t_{\mathrm{obs}}=30 \mathrm{~s}\right)\end{array}$ \\
\hline 40 & $\begin{array}{l}\quad 2 \kappa \Lambda \Delta_{1}^{2}=8.5( \pm 0.5) \mathrm{meV}^{2} \\
\tau_{1}=93( \pm 5) \mathrm{s}\end{array}$ & $\begin{array}{l}2 \kappa \Lambda \Delta_{1}^{2}=8.5( \pm 0.5) \mathrm{meV}^{2} \\
I_{0}=154( \pm 16) \mathrm{W} / \mathrm{cm}^{2}\end{array}$ \\
\hline 30 & $\begin{aligned} & 2 \kappa \Lambda \Delta_{1}^{2}=6.5 \mathrm{meV}^{2} \\
\tau_{1}= & 254( \pm 14) \mathrm{s}\end{aligned}$ & $\begin{array}{l}2 \kappa \Lambda \Delta_{1}^{2}=6.5 \mathrm{meV}^{2} \\
I_{0}=240( \pm 24) \mathrm{W} / \mathrm{cm}^{2}\end{array}$ \\
\hline 20 & $\begin{array}{l}2 \kappa \Lambda \Delta_{1}^{2}=4.6 \mathrm{meV}^{2} \\
\tau_{1}=274( \pm 19) \mathrm{s}\end{array}$ & $\begin{array}{l}2 \kappa \Lambda \Delta_{1}^{2}=4.6 \mathrm{meV}^{2} \\
I_{0}=326( \pm 19) \mathrm{W} / \mathrm{cm}^{2}\end{array}$ \\
\hline 10 & $\begin{aligned} & 2 \kappa \Lambda \Delta_{1}^{2}=2.9 \mathrm{meV}^{2} \\
\tau_{1}= & 278( \pm 9) \mathrm{s}\end{aligned}$ & $\begin{array}{l}2 \kappa \Lambda \Delta_{1}^{2}=2.9 \mathrm{meV}^{2} \\
I_{0}=305( \pm 11) \mathrm{W} / \mathrm{cm}^{2}\end{array}$ \\
\hline
\end{tabular}

the transient and not in the saturated value, then the role of the photon absorption is "catalytic," namely, to change the $\tau_{1}$ and not the $\kappa \Delta_{1}^{2}$ present in the expression for $\sigma^{2}(\infty)$. Further experiment can resolve this point, and so clarify the role of the excess photon energy on spectral diffusion. Equations (1b) and (3) imply that in the present model the excess energy acts by affecting $\tau_{k}$ and not the other terms. If, instead, it has an effect on $\sigma^{2}(\infty)=2 \kappa \Lambda \Delta_{1}^{2}$, the model would need to be modified to include an additional contribution to $2 \kappa \Delta_{1}^{2}$.

The parameters listed in Tables I and II were determined from the spectral diffusion data of Empedocles and Bawendi ${ }^{10}$ for CdSe nanoparticles with an average diameter of $57 \AA$. In the studies of the blinking statistics, excitation intensities up to several $\mathrm{kW} / \mathrm{cm}^{2}$ were often used, and so $\tau_{1}$ in the latter case could be much shorter than the values in Table I. Empedocles and Bawendi ${ }^{10}$ also observed that linewidth broadening varies among different QDs. Such heterogeneity may be due to a distribution of $Q_{0}$ among QDs or to other factors.

\section{B. Analysis of the blinking statistics data}

The experimental data of blinking statistics can be analyzed and fitted using Eq. (6a) and (6b) for $P(t)$. Shimizu

TABLE II. The fitted parameters $2 \kappa \Lambda \Delta_{1}^{2}$ and $J_{0}\left(J_{0}=\gamma_{F} \tau_{1,0} / 2 \sigma_{\text {abs }}\right)$ for Fig. 5(a) using $\sigma^{2}=2 \kappa \Lambda \Delta_{1}^{2}\left[1-\exp \left(-J / J_{0}\right)\right]$. The values of $2 \kappa \Lambda \Delta_{1}^{2}$ were obtained from Table I.

$40 \mathrm{~K}$

$2 \kappa \Lambda \Delta_{1}^{2}=8.5 \mathrm{meV}^{2}$ $J_{0}=4.3( \pm 0.2) \times 10^{4} \mathrm{~J} / \mathrm{cm}^{2}$

$30 \mathrm{~K}$

$2 \kappa \Lambda \Delta_{1}^{2}=6.5 \mathrm{meV}^{2}$ $J_{0}=8.9( \pm 0.3) \times 10^{4} \mathrm{~J} / \mathrm{cm}^{2}$

$20 \mathrm{~K}$

$2 \kappa \Lambda \Delta_{1}^{2}=4.6 \mathrm{meV}^{2}$ $J_{0}=11.0( \pm 0.1) \times 10^{3} \mathrm{~J} / \mathrm{cm}^{2}$

$10 \mathrm{~K}$

$2 \kappa \Lambda \Delta_{1}^{2}=2.9 \mathrm{meV}^{2}$

$J_{0}=9.4( \pm 0.1) \times 10^{3} \mathrm{~J} / \mathrm{cm}^{2}$

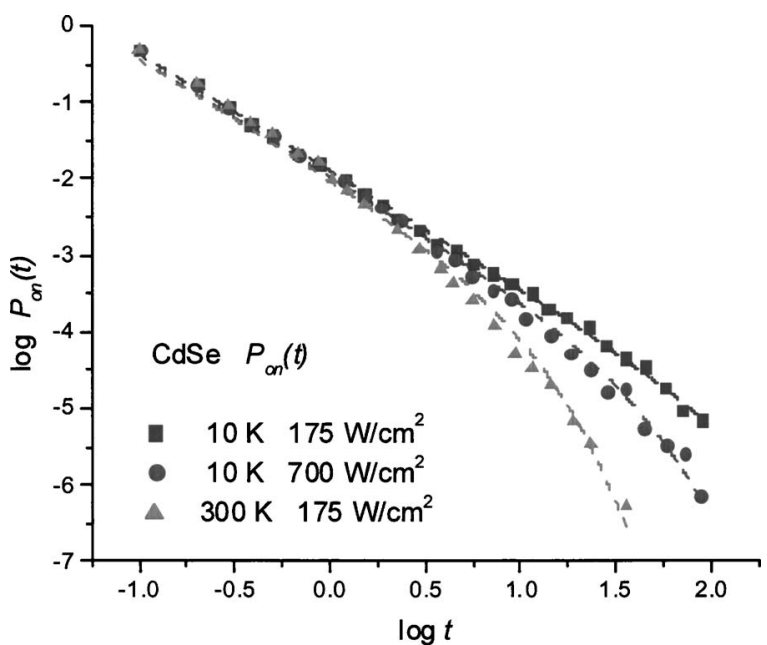

FIG. 6. The log-log plot of the time dependence of $P_{\text {on }}(t)$ for the "on" events from the experimental data of CdSe QDs by Shimizu et al. (Ref. 5) at three settings. Here $\log \left(P_{\text {on }}(t)\right)$ vs $\log (t)$ is used. The data were fitted to a power law with an exponential tail, i.e., $P_{\text {on }}(t) \sim t^{m} \exp (-\Gamma t)$ and the fitted parameters were shown in Table III.

et $a l^{5}$ observed a bending tail for $P_{\text {on }}(t)$ at higher temperatures or excitation intensities, but not for $P_{\text {off }}(t)$. This behavior agrees with the theoretical expressions for $\Gamma_{\text {on }}$ and $\Gamma_{\text {off }}$, and is, in the present model, principally due to the difference in $\tau_{1}$ and $\tau_{2}$. The data for $P_{\text {on }}(t)$ for CdSe QDs by Shimizu et $a l^{5}$ are replotted in Fig. 6 and fitted using $P_{\text {on }}(t)$ $\sim c t^{m} \exp$

$\left(-\Gamma_{\text {on }} t\right)$, where $c$ is an unimportant scaling constant, depending on how data were normalized. The fitted parameters are shown in Table III. The exponent $m$ of the power law is about $-1.53( \pm 0.02)$, using the data at $10 \mathrm{~K}$ and $175 \mathrm{~W} / \mathrm{cm}^{2}$ that show a simple straight line dependence in the log-log plot. The same exponent with $m \sim-1.53(0.02)$ was then kept fixed for two other curves to determine $\Gamma_{\text {on }}$ from the bending tail. The value of the exponent $m$ is very close to the ideal $-3 / 2$ of the present model with $P(t) \sim t^{-1.5} \exp \left(-\Gamma_{\text {on }} t\right)$. In the fitting of the bending tail for two other curves in Fig. 6 , instead of letting $m$ and $\Gamma$ float, $m$ was kept fixed at the same value as the first curve for better comparison among different $\Gamma_{\text {on }}$ 's (see Table III and footnote and the effect of fitting $m$ at -1.5 ).

In the present model, when the light intensity is increased four times from 175 to $700 \mathrm{~W} / \mathrm{cm}^{2}, 1 / \tau_{1}$ also increases four times, and so from the direct relationship between $\Gamma_{\text {on }}$ in Eq. (6a) and (6b), $\Gamma_{\text {on }}$ is expected also to increase by the same factor. Shimizu et al. ${ }^{5}$ observed a larger

TABLE III. The fitted parameters $m, \Gamma_{\text {on }}$, and $c$ for $P_{\text {on }}(t)$ of CdSe QDs in Fig. 6 at various experimental settings using $P_{\text {on }}(t)=c t^{m} \exp \left(-\Gamma_{\text {on }} t\right) . c$ in Tables III and IV are related to data normalization and can be different for each set of data. If $m$ was fixed at $-1.5,1 / \Gamma_{\text {on }}=121( \pm 16), 29( \pm 2)$, and $7.0( \pm 0.4)$, respectively.

\begin{tabular}{lclcc}
\hline \hline \multicolumn{1}{c}{$(\mathrm{K})$} & $\left(\mathrm{W} / \mathrm{cm}^{2}\right)$ & \multicolumn{1}{c}{$m$} & $\begin{array}{c}1 / \Gamma_{\text {on }} \\
(\mathrm{s})\end{array}$ & $c$ \\
\hline 10 & 175 & $-1.53( \pm 0.02)$ & $164( \pm 46)$ & $-1.90( \pm 0.01)$ \\
10 & 700 & -1.53 & $31( \pm 2)$ & $-1.96( \pm 0.03)$ \\
300 & 175 & -1.53 & $7.2( \pm 0.4)$ & $-1.97( \pm 0.04)$ \\
\hline \hline
\end{tabular}




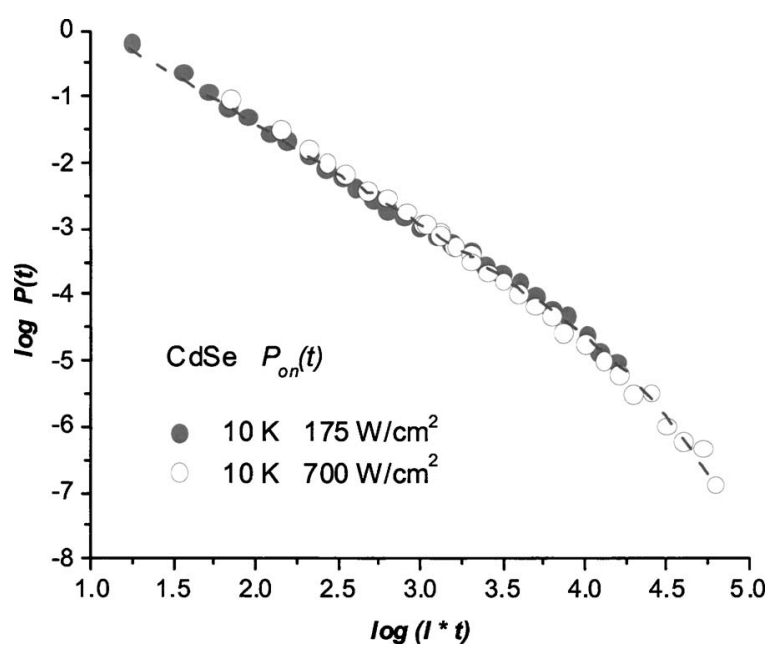

FIG. 7. The log-log plot of the energy density $(I \times t)$ dependence of $P_{\text {on }}(t)$ for the "on" events from the experimental data of CdSe QDs at $10 \mathrm{~K}$ and light intensity at both 175 and $700 \mathrm{~W} / \mathrm{cm}^{2}$. The data were best fitted to a power law with an exponential tail, i.e., $P_{\text {on }}(t)=a t^{-1.5} \exp (-I t / b)$ with $a$ $=1.58( \pm 0.01)$ and $b=2.04( \pm 0.09) \times 10^{3}$, where $a$ is a scaling constant dependent on how the data of $P(t)$ were normalized, and $1 / b=(\lambda$ $\left.+\Delta G^{0}\right)^{2} \sigma_{a b} / 4 \gamma_{F} \tau_{1} \Delta_{1}^{2}$. As also illustrated in Fig. 3(a), both spectral diffusion and intermittency data can be described by energy density as a universal variable.

bending with increased light intensity, a result which agrees with the fitted values of $1 / \Gamma_{\text {on }}$ in Table III at the two intensity settings. In addition, the bending also increased with the size of the QDs, which is consistent with the present model since $W$ increases with increasing surface area.

From the trend of the spectral diffusion data ${ }^{10}$ from 10 to $40 \mathrm{~K}$ in Table I, $\tau_{1}$ is expected to be much smaller at $300 \mathrm{~K}$ than at $10 \mathrm{~K}$. Since $\Gamma_{\text {on }}$ depends inversely on $\tau_{1}$ in Eq. (6a) and $(6 \mathrm{~b})$, the bending in the intermittency data should increase when temperature is increased, in agreement with the data.

In Eq. (6a) and (6b) with $P(t) \sim t^{-1.5} \exp \left(-\Gamma_{k} t\right), \Gamma_{k}$ is proportional to the light intensity, and one can use energy density $(I \times t)$ as a universal variable to describe the bending. As

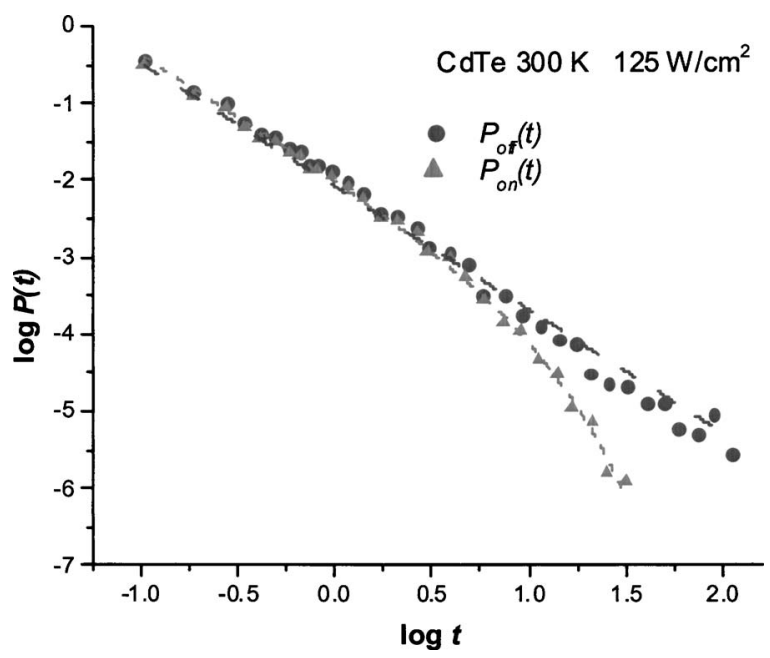

FIG. 8. The log-log plot of the time dependence of $P(t)$ for both "off" and "on" events from the experimental data of CdTe QDs at $300 \mathrm{~K}$ and $125 \mathrm{~W} / \mathrm{cm}^{2}$ by Shimizu et al. (Ref. 5). The data were fitted to $P_{\text {on }}(t)$ $\sim t^{m} \exp (-\Gamma t)$ and the fitted parameters were shown in Table IV.
TABLE IV. The fitted parameters $m, \Gamma$, and $c$ for $P_{\text {off }}(t)$ and $P_{\text {on }}(t)$ in Fig. 8 of CdTe QDs at $300 \mathrm{~K}$ and $125 \mathrm{~W} / \mathrm{cm}^{2}$ using $P_{\text {on }}(t)=c t^{m} \exp (-\Gamma t)$. In fitting $P_{\text {on }}(t)$ the exponent $m$ from $P_{\text {off }}(t)$ was used.

\begin{tabular}{clcc}
\hline \hline & \multicolumn{1}{c}{$m$} & $1 / \Gamma$ & $c$ \\
\hline$P_{\text {off }}(t)$ & $-1.6( \pm 0.1)$ & $\ldots$ & $-2.32( \pm 0.03)$ \\
$P_{\text {on }}(t)$ & -1.6 & $35( \pm 2) \mathrm{s}$ & $-2.05( \pm 0.02)$ \\
\hline \hline
\end{tabular}

noted earlier, this variable was used by Empedocles and Bawendi ${ }^{10}$ for spectral diffusion, and is suggested by Eq. (6a) and (6b) for the bending, $\Gamma$, also. The two experimental data sets of $P_{\text {on }}(t)$ at $10 \mathrm{~K}$ with light intensities of 175 and $700 \mathrm{~W} / \mathrm{cm}^{2}$ are plotted in Fig. 7 using energy density as a universal variable, and support the prediction.

The data for $P_{\text {off }}(t)$ and $P_{\text {on }}(t)$ for CdTe QDs by Shimizu et $a .^{5}$ at 10 and $300 \mathrm{~K}$ are plotted in Fig. 8 using the fitted parameters shown in Table IV. The exponent is also found to be close to the value of $-3 / 2$ for $\mathrm{CdSe}$. At $10 \mathrm{~K}$, the values of $\Gamma_{\text {on }}$ for CdTe (35 s) and CdSe (7 s) were obtained at different light intensities ( $\left.125 \mathrm{vs} 175 \mathrm{~W} / \mathrm{cm}^{2}\right)$. Even with the scaling factor adjusted for difference due to light intensity, a difference still exists, perhaps because of different compositions and sizes.

A bending tail of different form could also occur if the population sink occurs at the very bottom of the potential well, i.e., even with $\Gamma_{\text {on }}=0$. At a sufficiently longer time than the diffusion correlation time, the Green function approaches a constant, representing a system reaching equilibrium, and the power law also breaks down, even with $\Gamma_{\text {on }}=0$. A numerical evaluation of $P(t)$ with a population sink at the bottom of the well is illustrated in Fig. 9 and compared there with the experimental data of Shimizu et al. ${ }^{5}$ The calculated curves were obtained by numerical inversion of the Laplace transform in Eq. (A9a) and (A9b). As shown in the illustration, this kind of bending for activationless crossing follows the form of $\exp (-\Gamma t)$. This form does not fit well the experimental data that are best described by $t^{-1.5} \exp (-\Gamma t)$ of Eq. (6a) and (6b). Accordingly, the observed bending tail from the experiments that follow $t^{-1.5} \exp (-\Gamma t)$ supports the sce-

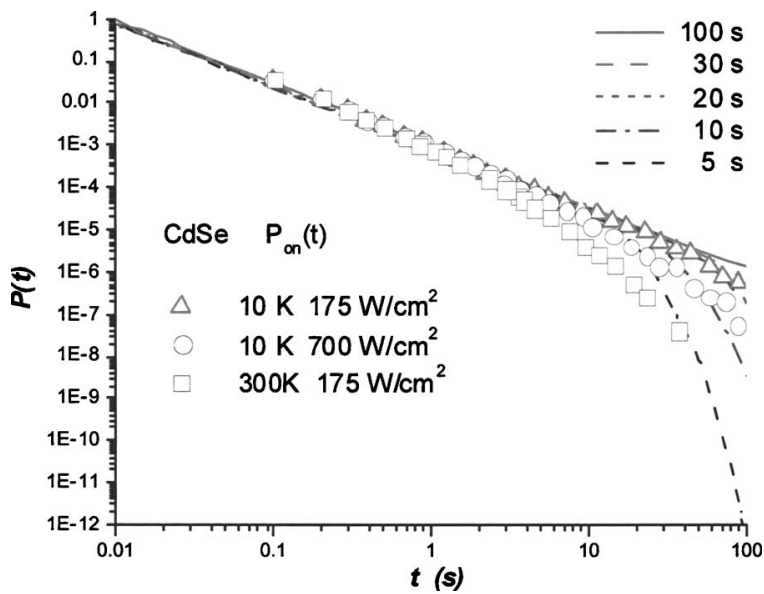

FIG. 9. Experimental $P(t)$ of Shimizu et al. (Ref. 5) for CdSe QDs, as compared with the calculated results for the special case of activationless crossing $\left(\lambda+\Delta G^{0}=0\right)$, which are best described by $\exp \left(-t / \tau_{1}\right)$ at $t$ comparable to $\tau_{1}$, and is different from the ideal form of $t^{-3 / 2} \exp (-\Gamma t)$. 
nario of energy-level crossing at a point other than the lowest point of the potential-energy well. When the crossing point occurs at the bottom of the potential-energy well, diffusion to the sink is not needed in the center for the wave packet to reach the crossing point, and a largely exponential decay ensues.

In some other reports ${ }^{6,8,9}$ on blinking statistics, a slightly steeper slope for the $\ln \left(P_{\text {on }}\right)$ vs $t$ was noted for the exponent of the power law. Fitting a straight line to a slightly bending curve could lead to a steeper slope, especially at higher light intensity. In principle, a larger exponent could occur by an anomalous diffusion process in the presence of a distribution of diffusion correlation times, as illustrated by Fig. 3(b) and discussed in Appendix C. Supplementing the previous intermittency study by Wang and Wolynes ${ }^{4}$ and a Lévy walk study of Barkai et al., ${ }^{42}$ more details and an extension of this DCET model to anomalous diffusion to accommodate the anomalies in the exponent will be published elsewhere.

\section{EXTRACTION OF MOLECULAR-BASED QUANTITIES AND FURTHER EXPERIMENTS}

A number of molecular-based quantities occur in the present analysis of spectral diffusion and intermittency in QDs. In the order of their appearance in various formulas, there are in Eqs. (1a), (1b), and (3) the diffusion coefficient $D_{1}$ for $\left|L^{*}\right\rangle$, the diffusion correlation time $\tau_{1}$ for $\left|L^{*}\right\rangle$, the Stokes shift $2 \Lambda$ between $\left|L^{*}\right\rangle$ and $|G\rangle$, the structural frequency $\Omega$, the diffusion coefficient $D_{2}$ in the dark state $|D\rangle$, the diffusion correlation time $\tau_{2}$, the reorganization energy for the charge transfer $\lambda$, the free-energy difference $\Delta G^{0}$ between $\left|L^{*}\right\rangle$ and $|D\rangle$, and the electronic coupling $V_{12}$.

Certain molecular-based parameters or combinations of them can be deduced from existing data. For example, in Table I $\tau_{1}$ and $2 \kappa \Lambda \Delta_{1}^{2}$ have been determined. From Eq. (3) and the vibration frequency $\hbar \Omega, \Lambda$ can be calculated from these data. Values are given in Tables III and IV for $\Gamma_{\text {on }}$, a compound quantity, $\left(\lambda+\Delta G^{0}\right)^{2} / 8 \tau_{1} \kappa \lambda \Delta_{1}^{2}$. At $10 \mathrm{~K}$ and $700 \mathrm{~W} / \mathrm{cm}^{2}$, with an estimate of $\tau_{1} \sim 35 \mathrm{~s}$, one has $(\lambda$ $\left.+\Delta G^{0}\right)^{2} / \kappa \lambda \Delta_{1}^{2} \sim 0.26$.

Further information could be obtained from the determination of $t_{c}$ if intermittency experiments at sufficiently short times become practical, and from an experiment to determine $\Gamma_{\text {off }}$, which may require temperatures and intensities which are perhaps too high to be realistic. Experiments to clarify the catalytic versus the saturation role of the excess photon energy were noted in Sec. IV. The recent experiment of Chung and Bawendi ${ }^{37}$ reports dark recombination reaction that occurs at a much longer time scale (several hours). At times much longer than the diffusion correlation time $\tau_{k}$, this model predicts that the system reaches equilibrium as the Green function approaches a constant, and deviation from power law is expected even for $\Gamma=0$. A full treatment of this DCET model at time beyond $100 \mathrm{~s}$ and slow charge recombination in the dark will be published elsewhere.

Another possible method to determine the electronic coupling is by electron paramagnetic resonance (EPR) techniques. The paramagnetic properties of the dark state can be elucidated from EPR experiments. ${ }^{43}$ Whether a QD is posi- tively or negatively charged, it depends on the physical properties of the surface state and traps. $|D\rangle$ represents the charge-separated dark state with a charge in a QD and a countercharge possibly trapped on the surface. In principle, a radical pair can exist in QDs, such as in the photoinduced radical pair ${ }^{44}$ found in photosynthetic systems. To avoid complications caused by quadrupolar interactions on EPR spin-echo modulations, QDs with nuclear spin greater than $1 / 2$ need to be avoided. The most abundant isotopes of $\mathrm{Cd}$, $\mathrm{Se}$, and Te have nuclear spins of $1 / 2$ or 0 and are good candidates. The hyperfine interactions from adjacent nuclear spins could cause the initial photoinduced singlet to evolve into a mixture of triplet and singlet, and the dipolar interaction of the electron-hole pair can manifest itself in the spinecho modulation. Further EPR experiments are needed to determine whether or not the triplet-singlet mixture is formed. The dipolar coupling and exchanger interaction between the radical pair can be measured by pulsed EPR techniques. ${ }^{45,46}$ These couplings can be used to determine the distance between the electron-hole pair and to estimate the electronic coupling element based on such a distance.

\section{ACKNOWLEDGMENTS}

We are pleased to acknowledge the support of the National Science Foundation and the Office of Naval Research. The authors would also like to acknowledge the support of one of the authors (J.T.) by the James W. Glanville Fellowship in Chemistry at the California Institute of California. One of the authors (R.A.M.) benefited considerably from the many interactions with Dr. Pavel Frantsuzov, and from calling his attention to several key articles. J.T. benefited from discussion with Ms. Chung and Prof. Bawendi on their recent work.

\section{APPENDIX A: STOCHASTIC LIOUVILLE EQUATION FOR THE PHOTOINDUCED DIFFUSION- CONTROLLED ELECTRON-TRANSFER (PIDCET) MODEL}

The stochastic Liouville equation ${ }^{21-25}$ for diffusioncontrolled electron-transfer reactions can be derived from the spin-boson model. ${ }^{23,25}$ In the master equation, the reaction coordinate in the electron-transfer processes in a polar environment is related to the change of electric displacement and the fluctuating dielectric polarization of the environment, or equivalent to the energy difference of the two electrontransfer states. With $\left|L^{*}\right\rangle$ denoted by 1 and $|D\rangle$ by 2 , one can apply such a stochastic Liouville formalism to these two states that are responsible for intermittency, and one has

$$
\begin{aligned}
\frac{\partial}{\partial t} \rho_{11}(Q, t)= & L_{11} \rho_{11}(Q, t)-\frac{2 \pi\left|V_{12}\right|^{2}}{\hbar} \delta\left(U_{1}(Q)-U_{2}(Q)\right) \\
& \times\left(\rho_{11}(Q, t)-\rho_{22}(Q, t)\right), \\
\frac{\partial}{\partial t} \rho_{22}(Q, t)= & L_{22} \rho_{22}(Q, t)-\frac{2 \pi\left|V_{21}\right|^{2}}{\hbar} \delta\left(U_{1}(Q)-U_{2}(Q)\right) \\
& \times\left(\rho_{22}(Q, t)-\rho_{11}(Q, t)\right) .
\end{aligned}
$$

The reaction coordinate $Q$ is assigned to describe the reac- 
tion between the photoexcited states $\left|L^{*}\right\rangle$ and $|D\rangle$. This coupled rate equation is needed if one wants to calculate the unconditional probability for an ensemble of QDs at time $t$ without a priori knowledge about whether a specific QD in that ensemble remains in light or dark, or whether it has gone through many blinking cycles during the whole time $t$. However, to calculate the blinking statistics for an "on" event of a single QD, one needs to determine the probability density $P(t)$ for a QD that stays continuously in $\left|L^{*}\right\rangle$ for a whole period of $t$ that exceeds the experimental bin time window of $0.1 \mathrm{~ms}$. The relevant rate equation to calculate such a conditional probability is the decoupled equation. This is equivalent to setting the population in the dark state to zero during the whole on period $t$. The relevant decoupled rate equation for $P(t)$ of a single neutral QD continuously staying in state $\left|L^{*}\right\rangle$ is the transition from $\left|L^{*}\right\rangle$ to $|D\rangle$ given by

$$
\frac{\partial}{\partial t} \rho_{11}(Q, t)=L_{11} \rho_{11}(Q, t)-\frac{2 \pi\left|V_{12}\right|^{2}}{\hbar \sqrt{2 \kappa_{E} \lambda}} \delta\left(Q-Q_{c}\right) \rho_{11}(Q, t),
$$

where

$$
L_{11}=D_{1}\left(\frac{\partial^{2}}{\partial Q^{2}}+\frac{1}{\Delta_{1}^{2}}\left(1+\left(Q+Q_{1}\right) \frac{\partial}{\partial Q}\right)\right), \quad D_{1}=\frac{\Delta_{1}^{2}}{\tau_{1}} .
$$

In Laplace transform notation we have

$$
\begin{aligned}
s \bar{\rho}_{11}(Q, s)-\delta\left(Q-Q_{c}\right)= & L_{11} \bar{\rho}_{11}(Q, s)-\frac{2 \pi\left|V_{12}\right|^{2}}{\hbar \sqrt{2 \kappa_{E} \lambda}} \delta(Q \\
& \left.-Q_{c}\right) \bar{\rho}_{11}(Q, s) .
\end{aligned}
$$

This equation describes diffusion on a parabola with a sink at the energy-level crossing, with the escape rate proportional to $\left|V_{12}\right|^{2}$ from the Golden rule. As noted in the text, the starting time for an on event begins by the hop from the dark state at the energy-level crossing at $Q=Q_{c}$ and starts its random-walk journey on the parabolic potential $U_{1}(Q)$ of the light state $\left|L^{*}\right\rangle$. The end time is when the population hops back to $|D\rangle$ at the sink at $Q=Q_{c}$. The initial population distribution is a delta-function distribution at the energy-level crossing point $Q_{c}$ and is normalized to unity.

For the "off" events, the relevant decoupled rate equation for $P(t)$ of the dark QD state continuously staying in state $|D\rangle$ is the transition from $|D\rangle$ to $\left|L^{*}\right\rangle$ given by

$$
\frac{\partial}{\partial t} \rho_{22}(Q, t)=L_{22} \rho_{22}(Q, t)-\frac{2 \pi\left|V_{21}\right|^{2}}{\hbar \sqrt{2 \kappa_{E} \lambda}} \delta\left(Q-Q_{c}\right) \rho_{22}(Q, t),
$$

where

$$
L_{22}=D_{2}\left(\frac{\partial^{2}}{\partial Q^{2}}+\frac{1}{\Delta_{1}^{2}}\left(1+Q \frac{\partial}{\partial Q}\right)\right), \quad D_{2}=\frac{\Delta_{2}^{2}}{\tau_{2}} .
$$

Because an electronically excited QD is either in $\left|L^{*}\right\rangle$ or $|D\rangle$ at any given time, to calculate the distribution of the duration period of a QD staying in a particular state $\left|L^{*}\right\rangle$ or $|D\rangle$ one only needs to use Eq. (A3) and (A4).

The distribution of the blinking statistics $P(t)$ for on events or off events is the rate of the loss for the total popu- lation in either the neutral or the charge-separated state. Using the Green function method, ${ }^{34}$ one can solve Eq. (A3) or (A4) with the Green function for the operators $L_{11}$ and $L_{22}$. From Eq. (A3) for the "light" state $\left|L^{*}\right\rangle$ one obtains

$$
\bar{\rho}_{11}(Q, s)=\bar{G}_{1}\left(Q, Q_{c} ; s\right)-\frac{2 \pi\left|V_{12}\right|^{2}}{\hbar \sqrt{2 \kappa_{E} \lambda}} \bar{G}_{1}\left(Q, Q_{c} ; s\right) \bar{\rho}_{11}\left(Q_{c}, s\right) .
$$

If we define $\bar{p}_{1}(Q, s)$ as the Laplace transform of the population in both $\left|L^{*}\right\rangle$ and $|G\rangle$ for the neutral (light) states, we have

$$
\bar{p}_{1}(Q, s)=\frac{\bar{G}_{1}\left(Q_{c}, Q_{c} ; s\right)}{1+\left(2 \pi\left|V_{12}\right|^{2} / \hbar \sqrt{2 \kappa_{E} \lambda}\right) \bar{G}_{1}\left(Q_{c}, Q_{c} ; s\right)} .
$$

A similar expression can be derived for $\bar{p}_{2}(Q, s)$ from Eq. (A4) for the dark states $|D\rangle$ and $\left|D^{*}\right\rangle$. One can show that the Laplace transform of $P_{k}(t)$, the distribution for the duration time $t$, is given by

$$
\begin{aligned}
\bar{P}_{k}(s) & =-\int_{0}^{\infty} d t e^{-s t} \frac{d}{d t}\left(\int_{-\infty}^{\infty} d Q p_{k}(Q, t)\right) \\
& =1-s \int_{-\infty}^{\infty} d Q \bar{p}_{k}(Q, s) \\
& =\frac{\left(2 \pi\left|V_{k}\right|^{2} / \hbar \sqrt{2 \kappa_{E} \lambda}\right) \bar{G}_{k}\left(Q_{c}, Q_{c} ; s\right)}{1+\left(2 \pi\left|V_{k}\right|^{2} / \hbar \sqrt{2 \kappa_{E} \lambda}\right) \bar{G}_{k}\left(Q_{c}, Q_{c} ; s\right)} .
\end{aligned}
$$

The analytical formula for the Green function in the time domain is well known and is given by ${ }^{27}$

$$
\begin{aligned}
G\left(Q, Q^{\prime} ; t\right)= & \frac{1}{\sqrt{2 \pi \Delta_{k}^{2}\left(1-\exp \left(-2 t / \tau_{k}\right)\right)}} \\
& \times \exp \left[-\frac{\left(\left(Q-x_{0, k}\right)-\left(Q^{\prime}-x_{0, k}\right) \exp \left(-t / \tau_{k}\right)\right)^{2}}{2 \Delta_{k}^{2}\left(1-\exp \left(-2 t / \tau_{k}\right)\right)}\right],
\end{aligned}
$$

where $x_{0, k}$ denotes the lowest point at the bottom of the $k$ th potential, i.e., $x_{0,1}=-Q_{1}$ and $x_{0,2}=0$. To calculate the blinking statistics given by Eq. (A7) one needs to evaluate the Laplace transform of $G_{k}\left(Q_{c}, Q_{c} ; t\right)$, which is given by

$$
\begin{aligned}
\bar{G}\left(Q, Q^{\prime}, s\right)= & \int_{0}^{\infty} d t \frac{1}{\sqrt{2 \pi \Delta_{k}^{2}\left(1-\exp \left(-2 t / \tau_{k}\right)\right)}} \\
& \times \exp \left[-s t-\frac{\left(Q_{c}-x_{0, k}\right)^{2}}{2 \Delta_{k}^{2}} \tanh \left(t / 2 \tau_{k}\right)\right] \\
\approx & \int_{0}^{\infty} d t \frac{\exp \left[-\left(s+\Gamma_{k}\right) t\right)}{\sqrt{4 \pi \Delta_{k}^{2}\left(t / \tau_{k}\right)}}=\frac{1}{\sqrt{4 \Delta_{k}^{2} / \tau_{k}}} \frac{1}{\sqrt{s+\Gamma_{k}}}
\end{aligned}
$$

where 


$$
\Gamma_{k} \equiv \frac{\left(Q_{c}-x_{0, k}\right)^{2}}{4 \tau_{k} \Delta_{k}^{2}} \equiv \frac{E_{a, k}}{2 \tau_{k} \kappa_{E} \Delta_{k}^{2}}, \quad E_{a, k} \equiv \frac{\left(\lambda \pm \Delta G^{0}\right)^{2}}{4 \lambda} .
$$

The potential-energy-surface crossing occurs at $Q_{c}=(\lambda$ $\left.-\Delta G^{0}\right) / \sqrt{2 \kappa \lambda}$, the bottom of the potential well of $U_{1}(Q)$ and $U_{2}(Q)$ are $x_{0,1}=Q_{1}, x_{0,2}=0$, respectively, and the electrontransfer reorganization energy is $\lambda=\kappa Q_{1}^{2} / 2$.

Equations (A7), (A9a), and (A9b) yield

$$
\bar{P}_{k}(s)=\frac{1}{1+\sqrt{\left(s+\Gamma_{k}\right) t_{c, k}}},
$$

and its inverse Laplace transform is

$$
P_{k}(t)=\frac{\exp \left(-\Gamma_{k} t\right)}{\sqrt{\pi t_{c, k}} t}\left[1-\sqrt{\frac{\pi t}{t_{c, k}}} \exp \left(\frac{t}{t_{c, k}}\right) \operatorname{erfc}\left(\sqrt{\frac{t}{t_{c, k}}}\right)\right],
$$

where $t_{c, k}$ equals $2 \kappa_{E} \lambda\left(\hbar \Delta_{k} / \pi V_{k}^{2} \sqrt{\tau_{k}}\right)^{2}$ as in Eq. (5c), and where $\operatorname{erfc}(x)$ is the complementary error function. Equation (A11) can be reduced to a simpler expression in two regimes as in Eq. (5a)-(5c) in the text.

In this work, the power law arises in the time regime shorter or comparable to the diffusion correlation time constant $\tau_{1}$. In another regime with fast diffusion (very short $\tau_{1}$ ) as occurs in most conventional electron transfer processes, the rate process becomes simple exponential where a different asymptote for the Green function needs to be used. ${ }^{23}$

To calculate the time dependence $P(t)$ of the blinking statistics from Eq. (A9a) and (A9b), the Laplace transform of the Green function in Eq. (A10) is evaluated numerically. The inverse Laplace transform algorithm was taken from IMSL. This numerical approach is limited by the accuracy in evaluating the Green function of Eq. (A9a) and (A9b) at small $s$ (long time). To calculate $P(t)$ for a wider range of time, one can directly solve Eq. (4a) or (4b) with the finiteelement method by replacing the differential equation by a difference equation in discrete lattice points. It is sufficient to use 5000 lattice points to represent the reaction coordinate $Q$ with an energy spacing about a thousandth of $\Delta$, and a time step of a hundredth of the inverse of $2 \pi\left|V_{k}\right|^{2} \hbar \sqrt{2 \kappa_{E} \lambda}$. Both approaches are equivalent and give similar results.

\section{APPENDIX B: FURTHER REMARKS ON $Q$ AND $Q$}

Earlier in treating spectral diffusion, it was pointed out that the reaction coordinate $q$ for spectral diffusion (associated with fluctuations of the energy difference between $\left|L^{*}\right\rangle$ and $|G\rangle)$ and the reaction coordinate $Q$ for electron transfer (associated with the energy difference between $\left|L^{*}\right\rangle$ and $|D\rangle$ ) are not the same. Considering time fluctuations in the energy difference between the electron states $\left|L^{*}\right\rangle$ and $|G\rangle$ that are linearly coupled to $N$-dimensional harmonic oscillators of the heat bath, the reaction coordinate $q$ represents the interaction energy as $q(t)=\sum_{k=0}^{N} c_{k}(t) q_{k}(t)$, where $q_{k}$ is the displacement for the $k$ th oscillator. On the other hand, for electron transfer between $\left|L^{*}\right\rangle$ and $|D\rangle$, the time fluctuation in their energy difference is represented by another reaction coordinate $Q$ with $Q(t)=\sum_{k=0}^{N} b_{k}(t) q_{k}(t)$. These two coordinates, as illus-
TABLE V. The exponent $m$ of the power law reported by various groups.

\begin{tabular}{ccc}
\hline \hline$m$ & Samples & References \\
\hline $\begin{array}{c}m_{\text {off }}=-1.5(10 \mathrm{~ms} / \mathrm{bin}) \\
m_{\text {off }}=-1.6(0.1 \mathrm{~ms} / \mathrm{bin})\end{array}$ & CdSe & 4 \\
$m_{\text {on }}, m_{\text {off }}=-1.5$ & CdSe & 5 \\
$m_{\text {on }}, m_{\text {off }}=-1.6$ & CdTe & \\
$m_{\text {on }}, m_{\text {off }}=-1.4$ & CdSe on gold substrate & 7 \\
$m_{\text {on }}=-1.6, m_{\text {off }}=-1.7$ & CdSe & 8 \\
$m_{\text {on }}=-1.58, m_{\text {off }}=-1.48$ & CdSe & 14 \\
$m=-1.65$ (autocorrelation method $)$ & CdSe & 9 \\
$m=-1.7$ (autocorrelation method $)$ & CdSe & 15 \\
$m_{\text {on }}=-2.0, m_{\text {off }}=-1.5$ & InP & 6 \\
$m_{\text {on }}=-2.2, m_{\text {off }}=-1.7$ & $\mathrm{Si}$ & 16 \\
\hline \hline
\end{tabular}

trated in Fig. 1(c), are not correlated because the interactions involve different pair of states and represent different projections from $N$-dimensional space. The observation of spectral diffusion corresponds to the projection onto $q$ space of the fluctuation in $N$-dimensional space. The intermittency of the fluorescence corresponds, instead, to a projection onto $Q$ space.

During the sampling period of $0.1 \mathrm{~s}$, the instrument integration time (bin time), immediately after the birth of the light state, a QD could have undergone many cycles between dark and light states, i.e., there are numerous returns to the $Q=0$ hyperplane. Each return is related to a change in the value of $q$, as discussed in the text.

\section{APPENDIX C: THE EXPONENT OF THE POWER LAW IN BLINKING STATISTICS}

In the ordinary diffusion discussed in Sec. II A, the diffusion constant $D$ is time-independent and the second moment grows in time if $t$ is much shorter than $\tau_{L}$, as shown in Eqs. (5a), (5b), (6a), and (6b) at very short times $P(t)$ $\sim t^{-1 / 2}$, at intermediate times $P(t) \sim t^{-3 / 2}$ and at later times $P(t) \sim t^{-3 / 2} \exp (-\Gamma t)$. The data for most experiments, illustrated in Figs. 5-8, follow a power law (with/without an exponential tail) with an exponent close to -1.5 . The exponent measured from various research groups is listed in Table V. A different exponent can arise in the continuous-time random-walk (CTRW) model of Montroll and Weiss. ${ }^{47}$ Such a model allows a distribution of the diffusion correlation time $\tau_{1}$, often used in studies of diffusion in solid with random trap distributions, invoke site-dependent hopping rate and time-dependent diffusion constant with non-Markovian process. By modifying the ordinary $1 \mathrm{D}$ diffusion with the CTRW model, or an anomalous diffusion with a timedependent diffusion constant, the power law for intermittency would not necessarily follow Eqs. (5a)-(5c), (6a), and (6b), and the spectral broadening in time could also differ from Eq. (3). An example is illustrated in Fig. 3(b), considering both the ordinary diffusion and anomalous diffusion. If the diffusion constant $D$ is time-dependent in anomalous dif- 
fusion, Eq. (A7) still applies except that the short-time behavior of the Green function can be different from the $t^{-1 / 2}$ dependence, depending on the distribution of the diffusion correlation time. $^{48}$ If the second moment of the displacement grows as $t^{\zeta}$ at short time in anomalous diffusion with $\zeta \neq 1$, the Green function has an asymptote at very small $t$ as $\sim t^{-\zeta / 2}$. According to Eq. (A9a) and (A9b), the blinking statistics $P(t)$ can have a different exponent for the power law. As illustrated in Fig. 3(b) for $\zeta=1$ and 1/2, at very short times $P(t) \sim t^{-\xi / 2}$ but becomes $t^{-2+\xi / 2}$ at longer times. The transition between these two regimes with a different exponent for the power law occurs at the critical time $t_{c}$ as illustrated in Fig. 3(b). The ideas of anomalous diffusion could be incorporated into the four-state model of the present work, if needed. Based on reasonably good fitting to various experimental data in Figs. 4-8, the present DCET model appears to be sufficient at this time. By extending this work to nonMarkovian diffusion ${ }^{25}$ with a distribution of the diffusion correlation time in the CTRW model, ${ }^{47,48}$ one could also accommodate the power law of a different exponent other than the ideal value of $-3 / 2$.

${ }^{1}$ W. J. Parak, T. Pellegrino, and C. Plank, Nanotechnology 16, R9 (2005).

${ }^{2}$ L. Jacak, P. Hawrylak, and A. Wojs, Quantum Dots (Springer, Berlin, 1997), and references therein.

${ }^{3}$ M. Nirmal, B. O. Dabbousi, M. G. Bawendi, J. J. Macklin, J. K. Trautman, T. D. Harris, and L. E. Brus, Nature (London) 383, 802 (1996).

${ }^{4}$ M. Kuno, D. P. Fromm, H. F. Hamann, A. Gallagher, and D. J. Nesbitt, J. Chem. Phys. 112, 3117 (2000).

${ }^{5}$ K. T. Shimizu, R. G. Neuhauser, C. A. Leatherdale, S. A. Empedocles, W. K. Woo, and M. G. Bawendi, Phys. Rev. B 63, 205316 (2001).

${ }^{6}$ M. Kuno, D. P. Fromm, H. F. Hamann, A. Gallagher, and D. J. Nesbitt, J. Chem. Phys. 115, 1028 (2001).

${ }^{7}$ K. T. Shimizu, W. K. Woo, B. R. Fisher, H. J. Eisler, and M. G. Bawendi, Phys. Rev. Lett. 89, 117401 (2002).

${ }^{8}$ M. Kuno, D. P. Fromm, S. T. Johnson, A. Gallagher, and D. J. Nesbitt, Phys. Rev. B 67, 125304 (2003).

${ }^{9}$ R. Verberk, A. M. van Oijen, and M. Orrit, Phys. Rev. B 66, 233202 (2002).

${ }^{10}$ S. A. Empedocles and M. G. Bawendi, J. Phys. Chem. B 103, 1826 (1999).

${ }^{11}$ Y. Jung, E. Barkai, and R. J. Silbey, Chem. Phys. 284, 181 (2002).

${ }^{12}$ C. A. Condat, Phys. Rev. A 39, 2112 (1989).

${ }^{13}$ G. L. Millhauser, E. E. Salpeter, and R. E. Oswald, Proc. Natl. Acad. Sci. U.S.A. 85, 1503 (1988).
${ }^{14}$ X. Brokmann, J. P. Hermier, G. Messin, P. Desbiolles, J. P. Bouchaud, and M. Dahan, Phys. Rev. Lett. 90, 120601 (2003).

${ }^{15}$ M. Pelton, D. G. Grier, and P. Guyot-Sionnest, Appl. Phys. Lett. 85, 819 (2004).

${ }^{16}$ F. Cichos, J. Martin, and C. von Borczyskowski, Phys. Rev. B 70, 115314 (2004).

${ }^{17}$ S. A. Empedocles and M. G. Bawendi, Science 278, 211 (1997).

${ }^{18}$ R. G. Neuhauser, K. T. Shimizu, W. K. Woo, S. A. Empedocles, and M. G. Bawendi, Phys. Rev. Lett. 85, 3301 (2000).

${ }^{19}$ V. Turck, S. Rodt, O. Stier, R. Heitz, R. Engelhardt, U. W. Pohl, D. Bimberg, and R. Steingruber, Phys. Rev. B 61, 9944 (2000).

${ }^{20}$ R. A. Marcus and N. Sutin, Biochim. Biophys. Acta 811, 265 (1985), and references therein for review of electron-transfer theory.

${ }^{21}$ L. D. Zusman, Chem. Phys. 49, 295 (1980).

${ }^{22}$ H. Sumi and R. A. Marcus, J. Chem. Phys. 84, 4894 (1986).

${ }^{23}$ I. Rips and J. Jortner, J. Chem. Phys. 87, 6513 (1987).

${ }^{24}$ Y. J. Yan, M. Sparpaglione, and S. Mukamel, J. Phys. Chem. 92, 4842 (1988).

${ }^{25}$ J. Tang, J. Chem. Phys. 104, 9408 (1996).

${ }^{26}$ M. Lax, J. Chem. Phys. 20, 1752 (1952).

${ }^{27}$ R. K. Pathria, Statistical Mechanics (Pergamon, Oxford, 1972).

${ }^{28}$ T. D. Krauss and L. E. Brus, Phys. Rev. Lett. 83, 4840 (1999).

${ }^{29}$ O. Cherniavskaya, L. Chen, and L. E. Brus, J. Phys. Chem. B 108, 4946 (2004).

${ }^{30}$ A. J. Williamson and A. Zunger, Phys. Rev. B 61, 1978 (2000).

${ }^{31}$ A. Franceschetti and A. Zunger, Phys. Rev. B 62, R16287 (2000).

${ }^{32}$ R. A. Marcus, Discuss. Faraday Soc. 29, 21 (1960).

${ }^{33}$ A. Warshel, J. Am. Chem. Soc. 86, 2218 (1982).

${ }^{34}$ P. M. Morse and H. Feshbach, Methods of Theoretical Physics (McGrawHill, New York, 1953).

${ }^{35}$ D. Morris, N. Perret, and S. Fafard, Appl. Phys. Lett. 75, 3593 (1999).

${ }^{36}$ S. Sanguinetti, K. Watanabe, T. Tateno, M. Wakaki, N. Koguchi, and M. Gurioli, Appl. Phys. Lett. 81, 613 (2002).

${ }^{37}$ I. Chung and M. G. Bawendi, Phys. Rev. B 70, 165304 (2004).

${ }^{38}$ S. W. Osborne, P. Blood, P. M. Smowto, Y. C. Xin, A. Stintz, D. Huffaker, and L. F. Lester, J. Phys.: Condens. Matter 16, S3749 (2004).

${ }^{39}$ M. Nirmal, D. J. Norris, M. Kuno, M. G. Bawendi, Al. L. Efros, and M. Rosen, Phys. Rev. Lett. 75, 3728 (1995).

${ }^{40}$ A. L. Efros and M. Rosen, Annu. Rev. Mater. Sci. 30, 475 (2000).

${ }^{41}$ J. Wang and P. Wolynes, J. Chem. Phys. 110, 4812 (1999).

${ }^{42}$ E. Barkai, Y. Jung, and R. Silbey, Annu. Rev. Phys. Chem. 55, 457 (2004)

${ }^{43}$ O. I. Micic, A. J. Nozik, E. Lifshitz, T. Rajh, O. G. Poluektov, and M. C. Thurnauer, J. Phys. Chem. B 106, 4390 (2002).

${ }^{44}$ J. Tang, M. C. Thurnauer, and J. R. Norris, Chem. Phys. Lett. 219, 283 (1994).

${ }^{45}$ S. A. Dzuba, P. Gast, and A. J. Hoff, Chem. Phys. Lett. 268, 273 (1997).

${ }^{46}$ J. Tang and J. R. Norris, J. Chem. Phys. 101, 5615 (1994).

${ }^{47}$ E. W. Montroll and G. H. Weiss, J. Math. Phys. 6, 167 (1965).

${ }^{48}$ M. Shlesinger, J. Stat. Phys. 10, 421 (1974). 